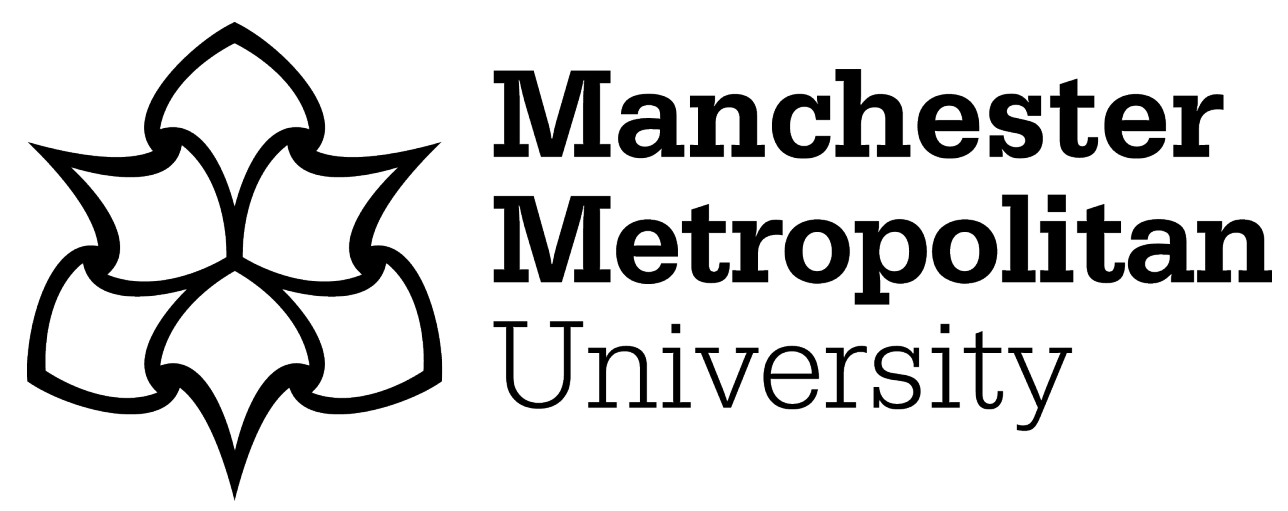

Clough, PJ ORCID logoORCID: https://orcid.org/0000-0002-7354-5445, Kalek, N, Gerber, M, Brand, S, Lemola, S, Sadeghi Bahmani, D, Pühse, U and Holsboer-Trachsler, E (2017) During early to mid adolescence, moderate to vigorous physical activity is associated with restoring sleep, psychological functioning, mental toughness and male gender. Journal of Sports Sciences, 35 (5). pp. 426-434. ISSN 0264-0414

Downloaded from: https://e-space.mmu.ac.uk/606080/

Version: Accepted Version

Publisher: Taylor \& Francis

DOI: https://doi.org/10.1080/02640414.2016.1167936

Please cite the published version 


\section{Mental toughness, sleep disturbances, and physical activity in patients with multiple sclerosis (MS) compared to healthy adolescents and young adults - a pilot study}

Dena Sadeghi Bahmani ${ }^{1}$, Markus Gerber ${ }^{2}$, Nadeem Kalak $^{1}$, Sakari Lemola ${ }^{3}$, Peter J. Clough ${ }^{4}$, Pasquale Calabrese ${ }^{5}$, Ludwig Kappos ${ }^{6}$, Edith Holsboer-Trachsler ${ }^{1}$, Serge Brand ${ }^{1,2}$

1 Psychiatric Clinics of the University of Basel, Center for Affective-, Stress- and Sleep

Disorders, Basel, Switzerland

2 Department of Sport, Exercise and Health, Sport Science Section, University of Basel, Basel, Switzerland

3 Department of Psychology, University of Warwick, Coventry, UK

4 Department of Psychology, Manchester Metropolitan University, Manchester, UK

5 Faculty of Psychology, Neuropsychology and Behavioral Neurology Unit, University of Basel, Basel, Switzerland

6 Department of Biomedicine, Neurology, University Hospital Basel, Basel, Switzerland

Address of correspondence:

Dena Sadeghi Bahmani, MSc

Psychiatric Clinics of the University of Basel

Center for Affective, Stress and Sleep Disorders

Wilhelm Klein-Strasse 27

4012 Basel - Switzerland

+41613255114 (voice)

+41613255 513 (fax)

dena.sadeghibahmani@upkbs.ch 


\section{Abstract}

Background: Multiple Sclerosis (MS) is the commonest chronic autoimmune demyelinating and inflammatory disease of the central nervous system, afflicting both body and mind. Typical symptoms are fatigue, paraesthesia and depression, along with cognitive impairments. Further, the risk to suffer from MS is 2.5 to 3.5-fold higher in females compared to males. Whereas there is extant research on fatigue, depression, and cognitive impairment of patients with MS during the clinical course, no research focused on sleep, psychological functioning, and physical activity at the time point of illness onset. The aims of the present study were therefore to assess possible state markers of mental toughness as a dimension of psychological functioning, sleep disturbances, physical activity among patients at the moment of illness onset, and to compare these data with those of healthy adolescents and healthy young adults.

Methods: A total of 23 patients with MS at illness onset (mean age: $\mathrm{M}=32.31$ years; $91 \%$ females), 23 healthy adolescents (mean age: $M=17.43$ years; $82 \%$ females), and 25 healthy young adults (mean age: $\mathrm{M}=20.72$ years; $80 \%$ females) took part in the study. They completed a booklet of questionnaires covering socio-demographic data, mental toughness, sleep disturbances, and physical activity.

Results: Patients with MS reported similar mental toughness traits as healthy adolescents and young adults, and an equal amount of moderate physical activity and sleep disturbances as young adults. Further, patients reported a lower level of vigorous physical activity, compared to both healthy adolescents and young adults.

Conclusions: Compared to healthy adolescents and young adults, patients with MS at illness onset reported similar mental toughness traits, sleep disturbances and levels of moderate physical 
activity. The pattern of results of the present pilot study suggests that the onset of MS is not predictable by poor mental toughness, poor sleep, and decreased moderate physical activity.

Keywords: multiple sclerosis, illness onset, mental toughness, sleep disturbances, physical activity, controls 


\section{Introduction}

Multiple Sclerosis (MS) is the commonest chronic autoimmune demyelinating and inflammatory disease of the central nervous system (CNS). Demyelination in association with axonal damage causing the slowness of nerve signals, leading in typical MS symptoms such as, feeling tired (fatigue), pain, visual problems, paresthesia, problems of movement and balance. ${ }^{1}$ Often, aftereffects such as depressive symptoms, social withdrawal, declined sexual drive, increased physical inactivity, ${ }^{2}$ and sleep disturbances are reported. ${ }^{3}$ Further, excessive fatigue severely reducing physical activity and exercising is experienced by at least two-thirds of people with MS. ${ }^{4}$

To treat MS during the illness course, in the meantime, a broad variety of medications are available, ${ }^{5-9}$ though, in the present paper this topic is not deepened, as we focused on psychological, sleep-, and physical activity-related characteristics at illness onset.

As regards fatigue, this is a core symptom of MS and understood as increased physical and psychological tiredness. Plausibly, fatigue might be associated with poor sleep, though, astonishingly, the association between poor sleep and fatigue has been understudied so far. Only recently, Strober ${ }^{10}$ reviewed the state-of-the-art and concluded that the association between sleep and fatigue has been largely understudied and unrecognized, and that poor sleep was a significant contributor to fatigue. We took this observation into account and, accordingly, the first aim of the present study was to assess, if and to what extent sleep disturbances might be a precursor of MS at illness onset, and to what extent these sleep disturbances were comparable to those of healthy adolescents and young adults. 
As regards physical activity, three main factors were identified to explaining why patients with MS are less active than healthy people: ${ }^{11,12}$ fatigue, impairment, and lack of time. Whereas lack of time seems to be a common reason for people not to exercise, impairment and fatigue are MS-specific issues. ${ }^{11-13}$ As regards impairment, there is some evidence of a negative feedbackloop in that acute PA may lead to immediate impairment and physical and mental discomfort. However, there is agreement that the impairments and discomfort are due to an acute increase in body temperature and not to PA as such (Uhthoff-phenomenon; cf. $^{14}$ ). Further, from the illness course of Huntington's Disease (HD), also a neurodegenerative disorder, we know that a lower active lifestyle has been considered a preclinical predictor of earlier illness onset. ${ }^{15}$ Thus, whereas it is well established that patients with MS during the course of illness report decreased physical activity, and whereas from another neurodegenerative disorder we learned that lower active lifestyle was associated with earlier illness onset, to the best of our knowledge, no data are available as regards the level of physical activity at illness onset among patients with MS. Accordingly, the next aim of the present study was to assess the level of moderate and vigorous physical activity of patients with MS at illness onset, and to compare these data with those from healthy adolescents and young adults.

Further, patients with MS, during the course of illness, reported increased symptoms of depression and anxiety ${ }^{16,17}$ while more recently, the psychological dimension of quality of life has gained further attention, thus shifting from a mere psychiatric point of view to a more comprehensive psychological status. ${ }^{18,19}$

While thus research on quality of life in patients with MS has gained increasing interest, we decided to introduce a further psychological dimension, that it, we asked about patients' status of mental toughness: Mental toughness (MT) is a concept characterized by an individual's 
capacity to be consistently successful in coping with difficult situations. ${ }^{20}$ Most studies in this field have been focused on MT in elite athletes; the results of those studies showed that mentally though athletes were able to cope with stress during a competition and to remain more focused and confident in case of a competition..$^{20-22}$

There are further studies discussing the association between MT and stress. For example, as Gerber el al..$^{23}$ showed that adolescents with higher scores of mental toughness were more resilient against stress. Another study showed that among adolescents, those who were mentally tougher, in case of stressful situation, were less at the risk of burnout, comparing to their peers with lower score in mental toughness. ${ }^{24}$ The bottom line of the results was that people with lower score in mental toughness reported higher scores in stress level while dealing with difficult and frustrating situations.

The concept of mental toughness has been recognized recently for its psychological importance, for example the application of MT in coping with stress, ${ }^{23,25}$ or its impact on both subjective ${ }^{26,27}$ and objective sleep quality, ${ }^{28}$ and accordingly, mental toughness has gained increasing interest due to its applicability on non-elite athletes such as healthy adolescents ${ }^{26-28}$, healthy young adolescents, ${ }^{25}$ lower and middle managers, senior managers, and clerical/administrative workers of early, middle and later adulthood. ${ }^{29}$ However, to the best of our knowledge, mental toughness traits have not been assessed so far in patients with MS, neither at illness onset, nor during the course of illness. A further aim of the present study was therefore to assess mental toughness traits among patients with MS at illness onset, and to compare these data with those from healthy adolescents and young adults.

Taken together, the concept of mental toughness has been proven to be associated with a broad variety of cognitive, emotional, and behavioral dimensions. For the following seven 
reasons, we introduced mental toughness in patients with MS: 1. Mental toughness consists of the four key factors Control (own life and emotions), Commitment, Challenge, and Confidence (in own abilities and in other people; see also Table 1), thus providing an excellent range of cognitive-emotional processes highly involved in coping with stress, emotions, unexpected events, and with social settings (confidence in other people). 2. Mental toughness is related to subjective and objective sleep. 3. Mental toughness is related to physical activity. 4. Mental toughness has not been assessed so far among patients with MS. 5. Mental toughness offers an excellent basis for both cross-sectional and longitudinal studies. 6. Mental toughness mirrors rather a trait, and not a state marker. 7. Given that the MTQ $48^{20}$ has been validated among different age and professional groups, data from further studies (samples) are comparable with new samples.

We took all these considerations into account and asked about the mental toughness profile of patients with MS at illness onset. Given that mental toughness is rather a trait marker, we holt that the scores did not vary immediately after a dramatic event such as the diagnose of MS. Further, we applied the MTQ48 ${ }^{20}$, and thus the data allow to comparison with previous research.

To summarize, the aims of the present study were to explore to what extent psychological dimensions (here: mental toughness), sleep disturbances, and physical activity might be deteriorated already at the onset of MS, thus providing indications of illness onset at a psychological, sleep-related and activity-related level. To do so, we assessed patients with MS at illness onset and compared their data with data from healthy adolescents and healthy young adults. We hold that the present pattern of results might be of practical importance, as until now, to the best of our knowledge, neurocognitive assessments to detect neurocognitive 
impairments, ${ }^{30-33}$ and imaging techniques to assess a cortical atrophy, ${ }^{34-38}$ seem to be the only reliable state markers to predict the onset of MS. These assessments, however, are time consuming, expensive, demand the inclusion of experts, and are not part of annual routine medical check-up. By contrast, changes in sleep quality, physical activity, and cognitive, emotional and behavioral frameworks are more easily detectable by people affected from MS and their close family members and friends.

For want of previous data of patients with MS at illness onset, instead of hypotheses, the following research questions were formulated: First, did mental toughness traits of patients with MS at illness onset statistically significantly differ from mental toughness traits of healthy adolescents and healthy young adults? Second, did patients with MS at illness onset report higher sleep disturbances, as compared to healthy adolescents and healthy young adults? Third, did patients with MS at illness onset report lower moderate and vigorous physical activity, as compared to healthy adolescents and healthy young adults?

\section{Method}

\section{Procedure}

Three different samples were assessed: Patients with MS at illness onset (Sample 1), healthy adolescents (Sample 2), and healthy young adults (Sample 3). Irrespective from the sample, all participants were informed about the voluntary character of participation. Further, they were informed that all data were gathered anonymously. Next, written and informed consent was given from participants or from their legal representatives in case of underage. All participants completed a booklet of questionnaires covering socio-demographic data, mental toughness, sleep disturbances and physical activity (see below). The local ethics committee approved the study, which has been performed in accordance of the rules laid down in the Declaration of Helsinki. 


\section{Samples}

Sample 1; patients with diagnosed multiple sclerosis (MS)

A total of 23 patients with diagnosed MS (mean age: $\mathrm{M}=32.31$ years; $\mathrm{SD}=7.04 ; 91.3 \%$ females) took part in the study. A trained neurologist not otherwise involved in the study diagnosed MS one to 30 days before patients were enrolled in the study. In other words: MS was diagnosed recently, and patients were new cases of MS. Patients with MS completed individually the questionnaire booklet during a routine check-up within 30-40 minutes. A trained psychologist assisted patients in completing the booklet in case of questions.

Sample 2: healthy adolescents

Data from 23 adolescents (mean age: $\mathrm{M}=17.43$ years; $\mathrm{SD}=1.91 ; 82.61 \%$ females) were taken from a larger data set and sample, as already largely described elsewhere (Brand et al, in press/2014). Briefly, participants were young adolescents from the catchment area of Basel (Basel, Switzerland) with regular schedules such as attending full time school, individual sports activity and leisure time activities such as playing music, attending music events, and similar. Of the full sample of 1475 adolescents, data of 23 participants were randomly selected to build up a subgroup for the present study (data set) and to represent the pattern of results of the entire sample (see Brand et al. in press/2014 for details). Participants needed about 35-45 min to complete the booklet during a regular school lesson.

Sample 3: healthy young adults

Data from 25 young adults (mean age: $\mathrm{M}=20.72$ years; $\mathrm{SD}=2.53 ; 80 \%$ females) were taken from a larger data set and sample, as already largely described elsewhere (Gerber et al., ${ }^{23}$ ). Briefly, participants were young adult students from the University of Basel (Basel, Switzerland), attending currently university lectures at full time. Of the full sample of 140 young adult students, data of 25 participants were randomly selected to build up a subgroup for the 
present study (data set) and to represent the pattern of results of the entire sample (see Gerber et al. ${ }^{23}$ for details). Participants needed about 25-30 min to complete the booklet after a lecture.

\section{Materials}

Participants completed a series of standardized questionnaires related to sociodemographic data, mental toughness (MT), sleep disturbances (SD), and physical activity (PA).

Socio-demographic background

Participants reported their gender, and age; additionally, patients with diagnosed MS reported the onset (days) of disease.

Mental toughness (MT)

To assess mental toughness, we used the Mental Toughness Questionnaire 48 (MTQ4820). It consists of 48 items, which are aggregated to the following dimensions: Challenge (e.g., "Challenges usually bring out the best in me"), commitment (e.g., "I don't usually give up under pressure"), emotional (e.g., "Even when under considerable pressure I usually remain calm”) and life control (e.g.," I generally feel in control"), and interpersonal confidence (e.g., "I usually take charge of a situation when I feel it is appropriate") and confidence in ability (e.g., "I am generally confident in my own abilities"). The factorial validity of MTQ48 has been proved in previous studies. Further, the MTQ48 has a high test-retest reliability and a high internal consistency. Items are anchored on 5-point Likert scales from 1 (= strongly disagree) to 5 (= strongly agree), with higher scores reflecting greater MT traits. Further, responses across items were summed to obtain an overall MT index (Cronbach's alpha=0.84-0.91).

Physical activity (PA) 
To assess physical activity, the short version of the International Physical Activity Questionnaire (IPAQ) was used. The IPAQ was developed by a working group initiated by the World Health Organization (WHO) and the Centers for Disease Control and Prevention. Based on the results from 12 countries, reliability and validity of IPAQ was comparable to other selfreported measures of PA. ${ }^{58}$ The short (self-administered, seven-item), last-week version of the IPAQ was administered, asking about the time spent for physical activity over the last 7 days. Minutes of sitting, walking, as well as moderate-intensity (walking not included) and vigorousintensity activities were calculated for the past week. The IPAQ questionnaires (short and long versions), including definitions of moderate and vigorous activity, are available at www.ipaq.ki.se.

Sleep disturbances (Insomnia Severity Index; ISI)

To assess sleep disturbances, the Insomnia Severity Index (ISI; Bastien, Vallieres, \& Morin, 2001) was used. This questionnaire is a seven-item self-rating screening measure for insomnia and an outcome measure for use in treatment research. The items, answered on 5-point rating scales ranging from 0 (= not at all) to 4 (= very much), refer, in part, to the Diagnostic and Statistical Manual of Mental Disorders (4th ed. [DSM-IV]; American Psychiatric Association, 2000) criteria for insomnia by assessing difficulty in falling asleep, difficulties remaining asleep, early morning awakenings, impaired daytime performance, impaired daytime performance, low satisfaction with sleep, and worry about sleep. The higher the overall score, the more the participant is assumed to suffer from insomnia (Cronbach's alpha $=.87$ ).

Statistical analysis

A Chi-square test was performed to compare gender distribution between the three groups. Next, a series of ANOVAs was performed with Group (patients with MS; healthy adolescents; healthy young adults) as independent factor and age, mental toughness, sleep disturbances, and physical activity as dependent variables. The nominal level of significance was 
set al alpha $<.05$. All statistical computations were performed with $\operatorname{SPSS} \circledast 22.0$ (IBM Corporation, Armonk NY, USA) for Apple Mac ${ }^{\circledR}$.

\section{Results}

Comparison of socio-demographic data between patients with MS, healthy adolescents, and healthy young adults

Table 1 reports all descriptive and inferential statistical indices; accordingly, statistical indices are not reported in the text anymore.

Age differed statistically significantly, with highest age for patients with MS, followed by healthy young adults, and healthy adolescents. Gender distribution was not significant.

Mental toughness scores, and sleep disturbances, separately for patients with MS, healthy adolescents, and healthy young adults

Table 2 shows all descriptive and inferential statistics of MT traits, separately for patients with diagnosed MS, healthy adolescents and healthy young adults; accordingly, statistical indices are not reported in the text anymore.

Significant mean differences were observed for challenge, commitment, control life, confidence in own abilities, for confidence total score, and for the MTQ48 total score A trend towards statistical significance $(\mathrm{p}<.1)$ was observed for Control total score.

Post-hoc analyses after Bonferroni-Holm corrections for p-values showed that compared to patients with MS and healthy adolescents, healthy young adults reported statistically significantly higher scores for challenge, confidence in own abilities, confidence total score and for the MTQ48 total score (Figure 1).

No statistically significant group differences were observed for control emotions and confidence interpersonal.

As regards sleep disturbances, statistically significant mean differences were observed. Post-hoc analyses after Bonferroni-Holm correction for p-values showed that healthy adolescents 
reported lowest sleep disturbances, whereas sleep disturbances did not differ between patients with MS and healthy young adults (Figure 2).

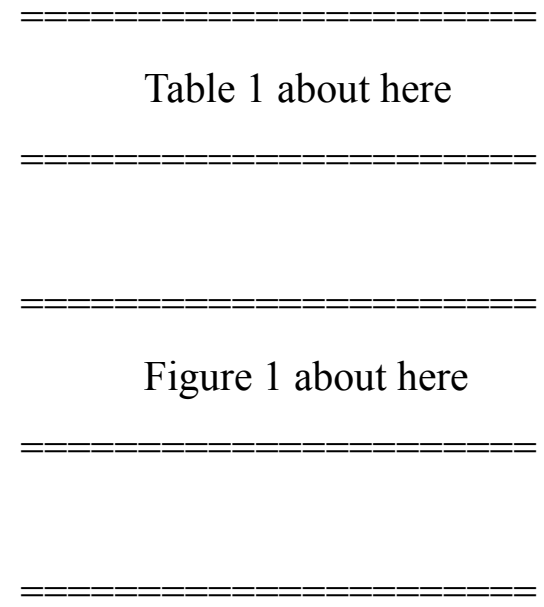

Figure 2 about here

Physical activity (PA), separately for patients with MS, healthy adolescents, and healthy young adults

Table 1 also reports all descriptive and inferential statistical indices related to physical activity.

Significant mean differences were observed for both moderate and vigorous PA. Post-hoc analyses after Bonferroni-Holm corrections p-values showed that young adults reported statistically significantly more vigorous PA compared to healthy adolescents and patients with MS, and that healthy adolescents reported more vigorous PA compared to patients with MS (see Figure 3). Further, patients with MS reported more moderate PA compared to healthy adolescents, and young adults reported more moderate PA compared to healthy adolescents, but not compared to patients with MS (see Figure 4).

Figure 3 about here 


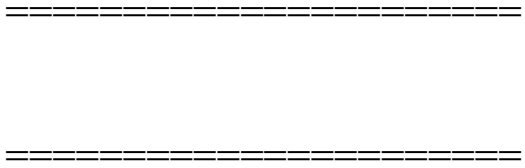

Figure 4 about here

\section{Discussion}

The key findings of the present study were that patients with MS at illness onset reported similar mental toughness traits as healthy adolescents and healthy adults, and an equal amount of moderate physical activity and sleep disturbances as young adults. Further, patients reported a lower level of vigorous physical activity, compared to both healthy adolescents and young adults. The present pattern of results adds to the current literature in that we were able to show that patients with MS at illness onset did not differ in mental toughness traits, sleep disturbances, and physical activity, compared to healthy adolescents and healthy young adults.

Three research questions were formulated and each of these is considered now in turn.

With the first research question we focused on possible differences in mental toughness traits between patients with MS at illness onset and healthy adolescents and young adults. The pattern of results showed a difference in the MT overall score compared to healthy young adults, but not compared to healthy 18 -years old healthy adolescents. Further, a deeper introspection (see Table 1) showed that patients with MS at illness onset did not statistically differ from healthy young adults as regards commitment, control life, control emotion, control overall score, and confidence interpersonal. Next, to further relativize the present results, we should take into account that the sample of healthy young adults consisted also of sports students, which almost by definition report a higher amount of mental toughness traits, ${ }^{39}$ along with a higher amount of physical activity.

Collectively, we claim that the present data add to the extant literature on mental toughness, in that this was the very first study on mental toughness traits among patients with MS at illness onset. Data do also add to the current literature on MS, in that the pattern of results suggests that at illness onset mental toughness traits are as high as those of healthy adolescents and young adults. Further and importantly, the present data are at odds with those studies reporting increased symptoms of depression and anxiety ${ }^{16,17}$ and a lower quality of life. ${ }^{18,19}$ 
With the second research question we asked if patients with MS at illness onset reported higher sleep disturbances, as compared to healthy adolescents and healthy young adults, and the answer was no. Therefore, again, we hold that the current pattern of results adds to the literature in that we were able to show that sleep at illness onset was not impaired, compared to healthy young adults. Accordingly, if this were the case, it could have been conceivable that either alterations in the neuronal centers responsible for sleep-wake-generation and coordination, ${ }^{40}$ or psychological issues (see Riemann et al. ${ }^{41}$ for the hyperarousal model of sleep disturbances), or both would have been responsible for decreased sleep. Further, the present results are at odds with those studies ${ }^{10,42,43}$ reporting impaired sleep patterns in patients with MS.

With the third research question we asked, if patients with MS at illness onset reported lower moderate and vigorous physical activity, as compared to healthy adolescents and healthy young adolescents, and the answer was no. Specifically, they reported a higher moderate physical activity, both compared to healthy adolescents, and, descriptively, compared to healthy young adults, while patients with MS at illness onset also reported a lower vigorous activity. Again, we hold that the present pattern adds to the current literature in that we were able to show that at illness onset, physical activity does not seem to be impaired or reduced. Likewise, and against the findings from patients with Huntington's Disease, ${ }^{15}$ physical activity patterns are not suitable as predictor of preclinical symptoms. Further, the present pattern of results is at odds with those studies showing a decreased physical activity among patients with MS. ${ }^{11,12,44}$

The novelty of the results should be balanced against the limitation of the study, which precludes an overgeneralization of the findings. First, the sample sizes were small, though, nevertheless, statistically significant mean differences were observed. Second, samples consisted prevalently of female participants, though, we note that females have a 2.5 to 3.5 -fold higher risk to suffer from MS, as compared to their male counterparts. Third, no objective data were assessed, and in this view, for instance objective physical activity assessments and objective sleep measurements would have allowed to get a more thorough picture and more reliable data of both physical activity and sleep. In this view, fourth, it would have been interesting and enlightening to compare the present data with neurocognitive performance and imaging data to compare to what extent MT traits, sleep disturbances, and physical activity were associated with neuronal changes and cognitive performance. Fifth, the present pattern of results might have emerged due to further latent, but unassessed dimensions, which might have biased two or more 
dimensions in similar or opposite directions. Sixth, it would have been interesting to assess daytime sleepiness and fatigue to associate these data with sleep disturbances. This would have allowed to investigating more thoroughly the association between poor sleep, tiredness and fatigue, given that the literature is sparse so far. Last, the cross-sectional character of the study does not allow to understanding the illness course; in this regard, it would be intriguing to know, if MT traits, sleep disturbances and the amount of physical activity might predict the future illness course, along with quality of life.

\section{Conclusions}

The pattern of results suggests that patients with MS at illness onset show comparable patterns of mental toughness traits, sleep disturbances, and physical activity as healthy adolescents and young adults. We further hold that the therapeutic challenge will be to keep these features stable over the time of treatment and illness.

\section{Acknowledgements}

We thank Nick Emler (University of Surrey, Surrey UK) for proofreading the manuscript.

\section{Conflict of interest}

All authors declare no conflicts of interests. The entire study has been performed without external funding. 


\section{References}

1. Noseworthy JH, Lucchinetti C, Rodriguez M, Weinshenker BG. Multiple sclerosis. N Engl J Med. 2000;343(13):938-952.

2. Svendsen KB, Jensen TS, Hansen HJ, Bach FW. Sensory function and quality of life in patients with multiple sclerosis and pain. Pain. 2005;114(3):473-481.

3. Veauthier C. Sleep disorders in multiple sclerosis. Review. Curr Neurol Neurosci Rep. 2015;15(5):21.

4. Branas P, Jordan R, Fry-Smith A, Burls A, Hyde C. Treatments for fatigue in multiple sclerosis: a rapid and systematic review. Health Technol Assess. 2000;4(27):1-61.

5. O'Connor P, Comi G, Freedman MS, et al. Long-term safety and efficacy of teriflunomide: Nine-year follow-up of the randomized TEMSO study. Neurology. 2016.

6. Lublin F, Miller DH, Freedman MS, et al. Oral fingolimod in primary progressive multiple sclerosis (INFORMS): a phase 3, randomised, double-blind, placebo-controlled trial. Lancet (London, England). 2016.

7. Kappos L, Wiendl H, Selmaj K, et al. Daclizumab HYP versus Interferon Beta-1a in Relapsing Multiple Sclerosis. N Engl J Med. 2015;373(15):1418-1428.

8. Kappos L, Radue EW, Chin P, Ritter S, Tomic D, Lublin F. Onset of clinical and MRI efficacy occurs early after fingolimod treatment initiation in relapsing multiple sclerosis. J Neurol. 2016;263(2):354-360.

9. Kappos L, Kuhle J, Multanen J, et al. Factors influencing long-term outcomes in relapsing-remitting multiple sclerosis: PRISMS-15. J Neurol Neurosurg Psychiatry. 2015;86(11):1202-1207.

10. Strober LB. Fatigue in multiple sclerosis: a look at the role of poor sleep. Frontiers in neurology. 2015;6:21.

11. Motl RW. Benefits, safety, and prescription of exercise in persons with multiple sclerosis. Expert Rev Neurother. 2014;14(12):1429-1436.

12. Asano M, Duquette $P$, Andersen R, Lapierre $Y$, Mayo NE. Exercise barriers and preferences among women and men with multiple sclerosis. Disabil Rehabil. 2013;35(5):353-361.

13. Lionetti E, Castellaneta S, Francavilla R, et al. Introduction of gluten, HLA status, and the risk of celiac disease in children. $N$ Engl J Med. 2014;371(14):1295-1303.

14. Waschbisch A, Tallner A, Pfeifer K, Maurer M. [Multiple sclerosis and exercise : effects of physical activity on the immune system]. Nervenarzt. 2009;80(6):688-692.

15. Trembath MK, Horton ZA, Tippett $L$, et al. A retrospective study of the impact of lifestyle on age at onset of Huntington disease. Movement 
disorders : official journal of the Movement Disorder Society. 2010;25(10):1444-1450.

16. de Cerqueira AC, Semionato de Andrade P, Godoy Barreiros JM, Teixeira $\mathrm{AL}$, Nardi AE. Psychiatric disorders in patients with multiple sclerosis. Compr Psychiatry. 2015;63:10-14.

17. Fiest KM, Walker JR, Bernstein CN, et al. Systematic review and metaanalysis of interventions for depression and anxiety in persons with multiple sclerosis. Multiple sclerosis and related disorders. 2016;5:1226.

18. Baumstarck K, Boyer L, Boucekine M, Michel P, Pelletier J, Auquier P. Measuring the quality of life in patients with multiple sclerosis in clinical practice: a necessary challenge. Multiple sclerosis international. 2013;2013:524894.

19. Baumstarck K, Boucekine M, Boyer L, et al. Quantification of relevance of quality of life assessment for patients with cognitive impairment: the suitability indices. BMC Neurol. 2014;14:78.

20. Clough P, Earle K, Sewell D. Mental toughness: The concept and its measurement. Solutions in sport psychology. 2002:32-43.

21. Mack MG, Ragan BG. Development of the mental, emotional, and bodily toughness inventory in collegiate athletes and nonathletes. J Athl Train. 2008;43(2):125-132.

22. Crust L, Clough PJ. Relationship between mental toughness and physical endurance. Percept Mot Skills. 2005;100(1):192-194.

23. Gerber M, Kalak N, Lemola S, et al. Are adolescents with high mental toughness levels more resilient against stress? Stress and Health. 2013;29(2):164-171.

24. Gerber $M$, Feldmeth AK, Lang $C$, et al. THE RELATIONSHIP BETWEEN MENTAL TOUGHNESS, STRESS, AND BURNOUT AMONG ADOLESCENTS: A LONGITUDINAL STUDY WITH SWISS VOCATIONAL STUDENTS (.). Psychological reports. 2015;117(3):703-723.

25. Gerber M, Brand S, Feldmeth AK, et al. Adolescents with high mental toughness adapt better to perceived stress: A longitudinal study with Swiss vocational students. Personality and Individual Differences. 2013;54(7):808-814.

26. Brand S, Kalak N, Gerber M, et al. During early and mid-adolescence, greater mental toughness is related to increased sleep quality and quality of life. J Health Psychol. 2014.

27. Brand S, Gerber M, Kalak N, et al. "Sleep Well, Our Tough Heroes!"-In Adolescence, Greater Mental Toughness is Related to Better Sleep Schedules. Behavioral sleep medicine. 2014;12(6):444-454.

28. Brand S, Gerber M, Kalak N, et al. Adolescents with greater mental toughness show higher sleep efficiency, more deep sleep and fewer awakenings after sleep onset. Journal of Adolescent Health. 2014;54(1):109-113. 
29. Perry JL, Clough PJ, Crust L, Earle K, Nicholls AR. Factorial validity of the mental toughness questionnaire-48. Personality and Individual Differences. 2013;54(5):587-592.

30. Tiemann L, Penner IK, Haupts M, Schlegel U, Calabrese P. Cognitive decline in multiple sclerosis: impact of topographic lesion distribution on differential cognitive deficit patterns. Mult Scler. 2009;15(10):11641174.

31. Lensch E, Matzke M, Petereit HF, Scherer P, Schramm S, Calabrese P. Identification and management of cognitive disorders in multiple sclerosis--a consensus approach. J Neurol. 2006;253 Suppl 1:129-31.

32. Calabrese P. Neuropsychology of multiple sclerosis--an overview. J Neurol. 2006;253 Suppl 1:I10-15.

33. Kalbe E, Calabrese P, Fengler S, Kessler J. DemTect, PANDA, EASY, and MUSIC: cognitive screening tools with age correction and weighting of subtests according to their sensitivity and specificity. Journal of Alzheimer's disease : JAD. 2013;34(4):813-834.

34. Calabrese M, Gajofatto A, Gobbin F, et al. Late-onset multiple sclerosis presenting with cognitive dysfunction and severe cortical/infratentorial atrophy. Mult Scler. 2015;21(5):580-589.

35. Calabrese M, Favaretto A, Poretto V, et al. Low degree of cortical pathology is associated with benign course of multiple sclerosis. Mult Scler. 2013;19(7):904-911.

36. Calabrese M, Favaretto A, Martini V, Gallo P. Grey matter lesions in MS: from histology to clinical implications. Prion. 2013;7(1):20-27.

37. Calabrese $\mathrm{M}$, Atzori $\mathrm{M}$, Bernardi $\mathrm{V}$, et al. Cortical atrophy is relevant in multiple sclerosis at clinical onset. J Neurol. 2007;254(9):1212-1220.

38. Calabrese M, Agosta F, Rinaldi F, et al. Cortical lesions and atrophy associated with cognitive impairment in relapsing-remitting multiple sclerosis. Arch Neurol. 2009;66(9):1144-1150.

39. Gerber M, Kalak N, Lemola S, et al. Adolescents' exercise and physical activity are associated with mental toughness. Mental Health and Physical Activity. 2012;5(1):35-42.

40. Cirelli C, Tononi G. Molecular neurobiology of sleep. Handbook of clinical neurology. 2011;98:191-203.

41. Riemann D, Spiegelhalder K, Feige B, et al. The hyperarousal model of insomnia: a review of the concept and its evidence. Sleep medicine reviews. 2010;14(1):19-31.

42. Strober LB, Arnett PA. An examination of four models predicting fatigue in multiple sclerosis. Archives of clinical neuropsychology : the official journal of the National Academy of Neuropsychologists. 2005;20(5):631-646.

43. Arnett PA, Strober LB. Cognitive and neurobehavioral features in multiple sclerosis. Expert Rev Neurother. 2011;11(3):411-424.

44. Razazian N, Yavari Z, Farnia V, et al. Exercising Impacts on Fatigue, Depression, and Paresthesia in Female Patients with MS. Medicine and science in sports and exercise. 2015. 
